\title{
Small bowel enterocyte abnormalities caused by methotrexate treatment in acute lymphoblastic leukaemia of childhood
}

\author{
NJT GWAVAVA, CR PINKERTON, JFT GLASGOW, JM SLOAN, JM BRIDGES \\ From the Departments of Child Health and Pathology, Institute of Clinical Science, Grosvenor Road, \\ Belfast BT12 6BJ
}

SUMMARY Jejunal biopsy specimens from 10 children with acute lymphoblastic leukaemia on methotrexate treatment were compared with 10 from children being investigated for diarrhoea o옹 failure to thrive. In association with methotrexate treatment on both light and electron microscopy $\vec{\imath}$ there were marked morphological abnormalities in the villus enterocytes. These consisted of striking distention of the lateral basal intercellular spaces, cell vacuolation and patchy necrosis and was most marked when methotrexate treatment was given between 24 and $72 \mathrm{~h}$ before biopsy. Tw $\infty$ mechanisms may be involved: an early direct toxic effect on the mature enterocyte coupled with interference with crypt cell generation, possibly causing ageing and loss of cells proximal to the normal extrusion zone. These pathological changes may account for the malabsorption in association? with methotrexate treatment.

Farber's first description of the use of folate antagonists in the management of acute lymphoblastic leukaemia (ALL), ${ }^{1}$ emphasised the limitations of treatment because of intestinal toxicity such as gastrointestinal ulceration and haemorrhage. ${ }^{2}$ Although such severe side effects are now less common they remain an important potential hazard. Xylose malabsorption has been reported in association with methotrexate (MTX) treatment in children with $\mathrm{ALL}^{3}$ and it has been suggested that the absorption of the drug itself may be similarly impaired. 45 This may contribute to the wide variations in serum MTX concentrations after a standard oral dose. ${ }^{467}$ Some children achieve only very low concentrations, therefore, which may be associated with a suboptimal response to treatment.

As part of a detailed study of factors influencing MTX absorption, jejunal biopsies were carried out in association with routine bone marrow aspiration. This paper describes striking abnormalities in the jejunal mucosal cells both on light and electron microscopy.

\section{Patients and methods}

Twenty jejunal biopsy specimens were studied; $1 \%$ were from children with ALL receiving MTX treatment whose ages ranged from 2.6 to $12.5 \mathrm{yr}$ and 10 were from sequential patients being investigate for diarrhoea or failure to thrive or both (controb group). The children with ALL were receiving treatment according to the Medical Research Council's Working Party on Childhood Leukaemiz (UK ALL Trials); the time since diagnosis was3 months to 5 yr. At biopsy, all were in clinicaf remission as determined by peripheral blood and bone marrow examination and in a good nutritionaR state; none had clinical alimentary toxicity, nof biochemical evidence of folate deficiency. They hack received oral MTX, $15 \mathrm{mg} / \mathrm{m}^{2}$, but its timing and the number of doses in relation to jejunal biopsy varied (Table). 
Relation of methotrexate treatment to villus enterocyte damage and morphometry

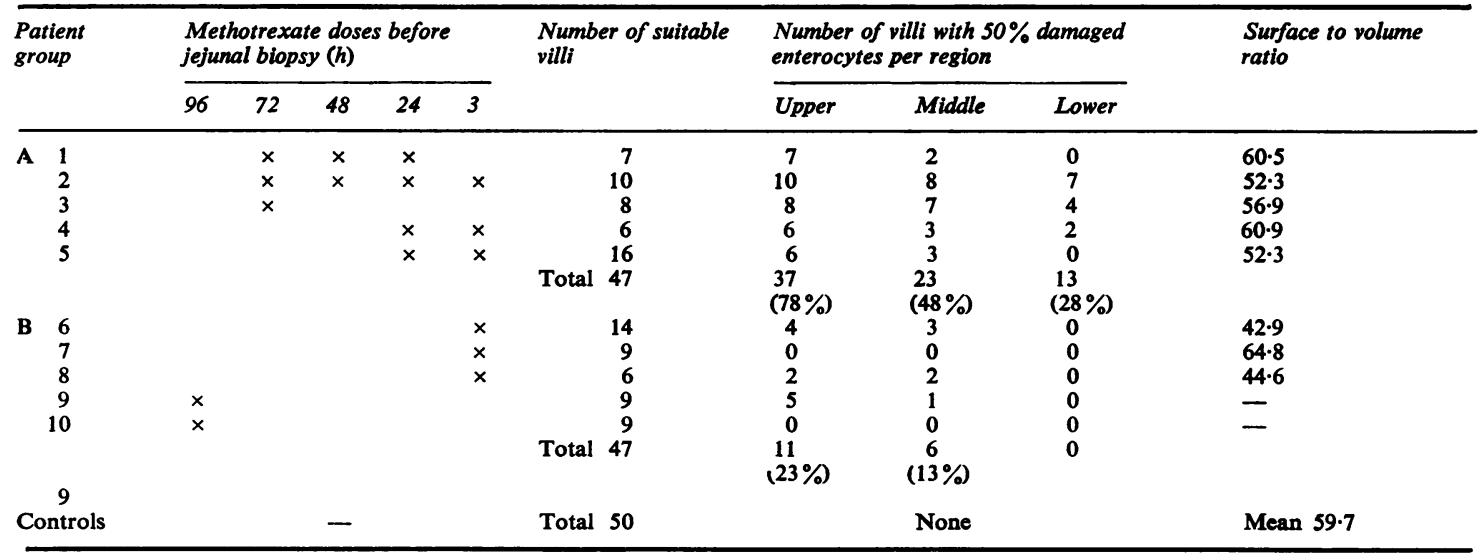

The specimen, taken close to the ligament of Treitz, was orientated under a Wild stereo-microscope and halved for both light and transmission electron microscopy. The study protocol received the approval of the Ethical Committee of the Faculty of Medicine of The Queen's University of Belfast.

\section{HISTOPATHOLOGY}

One specimen was fixed in Susa solution overnight, embedded in paraffin and stained with haematoxylin and eosin ( $\mathrm{H}$ and $\mathrm{E})$ for routine tissue morphology. A second specimen was placed for $4 \mathrm{~h}$ in Karnovsky's fixative containing $2.5 \%$ glutaraldehyde and $4 \%$ paraformaldehyde in $0.1 M$ phosphate buffer at pH 7.4. The specimen was stored in $0 \cdot 1 M$ phosphate buffer containing $0 \cdot 1 M$ sucrose at $\mathrm{pH} 7 \cdot 4$ after six washes in the same buffer. Post fixation was carried out for $2 \mathrm{~h}$ in 2 parts of osmium tetroxide to 1 part of $0.2 M$ Collidine buffer. After washing, the specimen was dehydrated in alcohol and passed through propylene oxide to fresh Spurr-resin embedding medium and dust-free gelatin capsules. After incubating the blocks at $60^{\circ} \mathrm{C}$ overnight, $1 \mu \mathrm{m}$ semithin sections were cut and stained with toluidine blue for detailed light microscopic examination. Morphometric examination and calculation of the surface to volume ratio (SVR) was carried out with the aid of a Weibel graticule (line length $1.8 \mathrm{~mm}$ ) placed in the microscope eyepiece as previously described. 89 To quantify the extent and distribution of morphological abnormalities, each villus was divided into three equal lengths - the upper, middle and lower regions, measured from the villus tip to the crypt mouth.10 In each region, the number of villi having greater than $50 \%$ damaged enterocytes was counted and expressed as a percentage of the total number of suitably orientated villi.
Ultrathin sections $(50-100 \mathrm{~nm})$ were cut from selected areas using an LKB Ultra-tome 111. These were lifted off copper grids and double-stained with uranyl acetate followed by lead citrate. The sections were then examined with an AEl EM 801 transmission electron microscope.

\section{Results}

The majority of biopsy specimens from children receiving MTX treatment showed no distinct abnormalities with $\mathrm{H}$ and $\mathrm{E}$ staining. The semithin sections stained with toluidine blue showed greater cellular detail and revealed several alterations not seen with $\mathrm{H}$ and $\mathrm{E}$ (Fig. 1). These abnormalities were most striking in those patients who had received MTX between 24 and $72 \mathrm{~h}$ before biopsy (group A).

On light microscopy cellular atrophy was evident in the upper and middle regions of villi with marked enlargement of the lateral basal intercellular spaces (LBIS) (Fig. 1). Many cells showed vacuolar degeneration associated with reduced staining affinity. In two cases areas of villus epithelium were separated from the basement membrane to produce small blebs. The nature of cellular abnormalities varied widely both between patients and within individual villi. In some, distention of the LBIS was the dominant abnormality, whereas in others cellular degeneration and vacuolation were prominent.

Semi-quantitative assessment of the biopsy specimens indicated a relation between the severity of the lesions and the time since MTX treatment, up to $72 \mathrm{~h}$ before biopsy (Fig. 2). Similarly, but to a lesser extent, there was an association with the total dose of MTX given before biopsy. In group A biopsies, the enterocytes in the upper part of the villi 


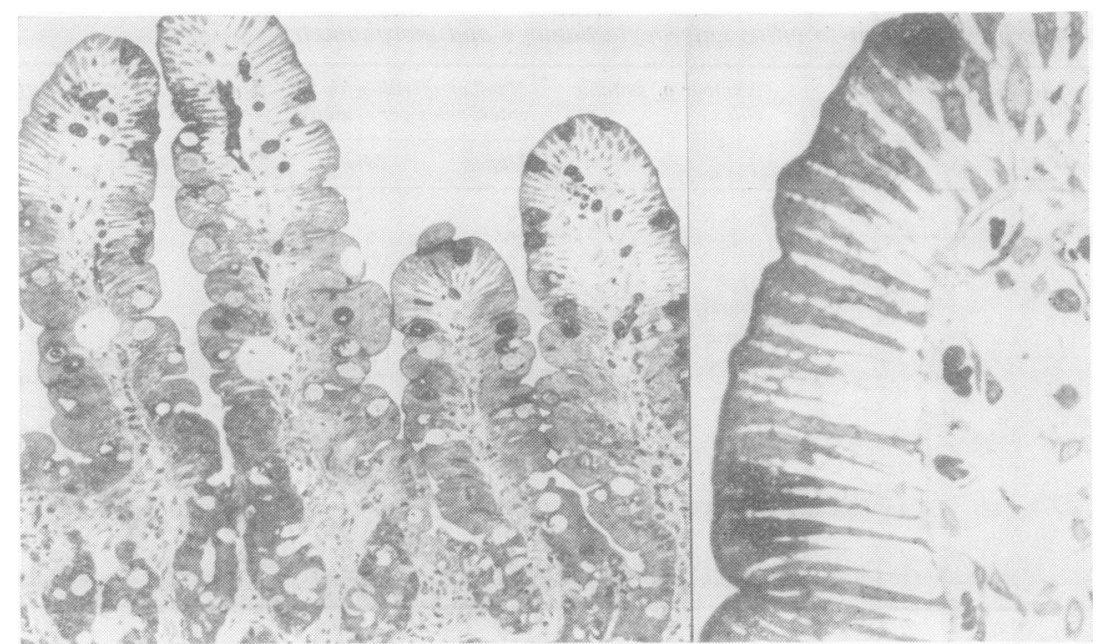

Fig. 1 Semithin section of jeiunum $72 \mathrm{~h}$ after single dose of methotrexate (patient 3 ). Villus architecture is preserved but clefts are seen between shrunken enterocytes in the upper and middle regions which also appear somewhat swollen.

Toluidine blue $\times 100$; and $\times 400$ magnification of a single villus from the same patient.

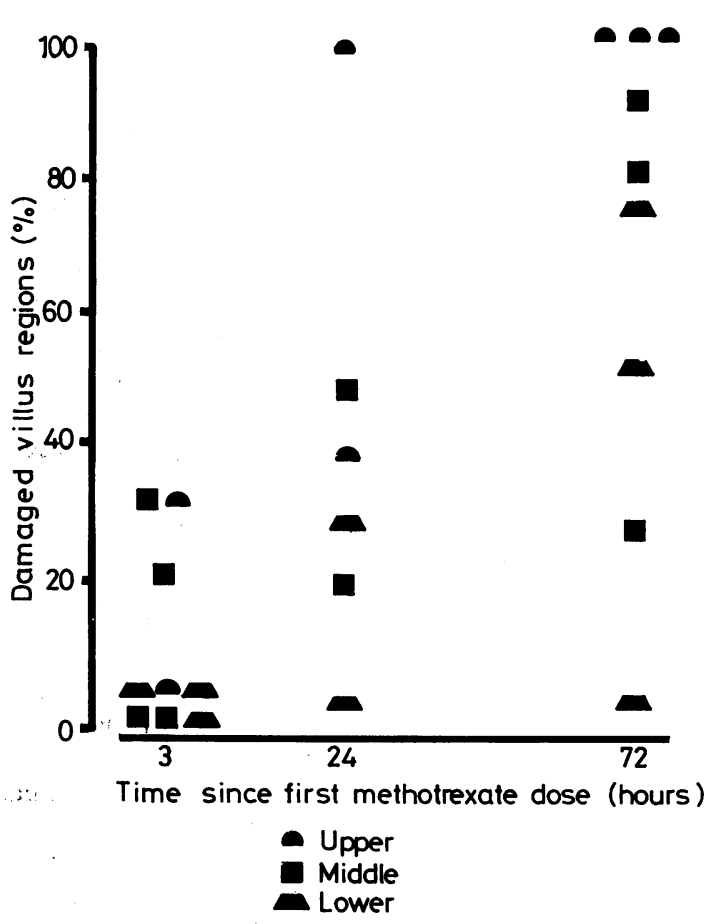

Fig. 2 Relation between the time of the first dose of methotrexate and damage in the upper, middle, and lower regions of the villus; the number of damaged regions (as defined by the Table) are expressed as a percentage of the total number of suitable villi counted. showed more damage than in those of patients who had received a single dose either very recentlys (within $3 \mathrm{~h}$ ) or $96 \mathrm{~h}$ before biopsy (group B) (Table)응 There was a similar difference in the middle region but damage to enterocytes in the lower region of the villi was evident only in the specimens of group $A \overrightarrow{\vec{B}}$ patients. Overall, there was a significantly higher 3 percentage of damaged villi in group $\mathbf{A}$ than in group? B biopsy specimens $(p<0.01)$. Nine of the controp. specimens showed normal villus architecture with both $\mathrm{H}$ and $\mathrm{E}$ and toluidine blue stains. A specimen from one patient showed the abnormalities character 3 istic of coeliac disease and has therefore been excluded from further reference. There was no significant difference between the SVR of specimens of MTX-treated children (groups A and B) and the nine controls.

On electron microscopy the most marked changes were again seen in the specimens of children who hados received MTX 24 to $72 \mathrm{~h}$ previously. Ultrastructurah abnormalities involved the microvilli, cell membranes, and cytoplasmic organelles. The electron micro- $-\frac{\omega}{\sigma}$ graphs shown are of abnormalities present in cells in the middle regions of villi. In damaged enterocyte? the microvilli were uneven, shortened and looselyes packed (Fig. 3). In some cells, more marked changes were apparent with loss of staining propertiesvacuolation and atrophy.

There was marked enlargement of the LBIS $\frac{\rho}{\mathbb{P}}$ which were wedge-shaped, the apex being at the mucosal surface and the base toward the basement 


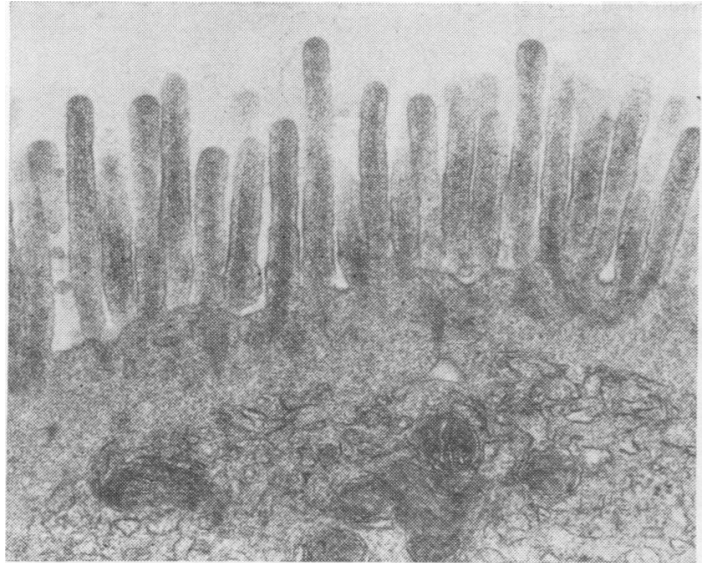

Fig. 3 Irregular and loosely packed microvilli of a methotrexate-damaged enterocyte in patient 2. EM $\times$ 20000.

membrane. These spaces were traversed at various points by delicate strands of cytoplasm extending from neighbouring cells and meeting at a desmosome.
Most enterocytes remained in contact with the basement membrane, which was itself preserved (Fig. 4). Intercellular contact was also maintained along the basement membrane in most areas, although in some there was complete separation of cells.

There was a marked reduction in the hyaloplasmic bulk with relative crowding of organelles, particularly mitochondria. In some cells focal dilatation of endoplasmic reticulum and Golgi apparatus was noted and the resulting cystic spaces gave rise to a degenerative vacuolar appearance (Fig. 5). In some villi single enterocytes or clusters of two or three cells were necrotic with swollen electrondense mitochondria and little internal structure discernible (Fig. 5). Apart from some degree of elongation, the nuclear structure was generally preserved, except in necrotic cells, where nuclei were pyknotic and electron-dense.

In contrast, the villi of patients in group B, who had received a single dose of MTX generally showed less marked changes which when present were patchy in their distribution. Microvilli were more regular and

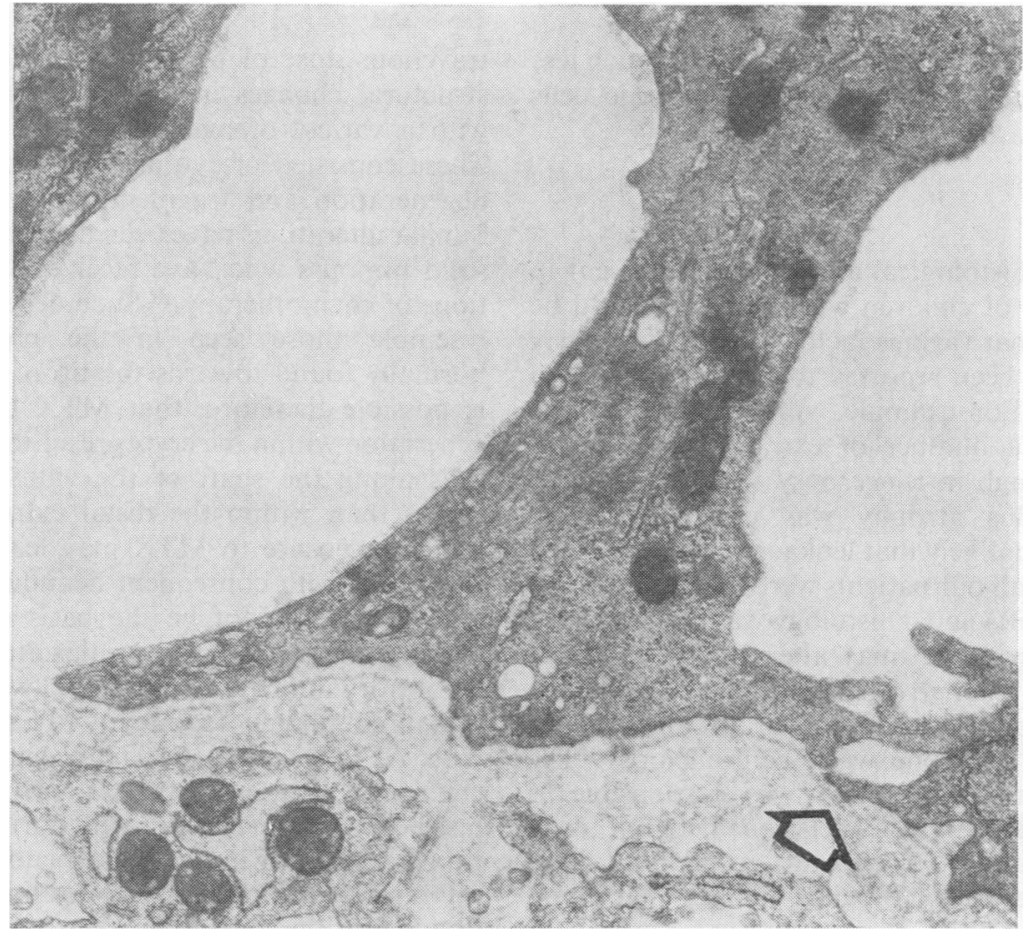

Fig. 4 Electromicrograph showing the basal region of damaged enterocytes. Marked enlargement of the lateral basal intercellular spaces is seen but intercellular contact between two cells is maintained at the basement membrane (arrow) in patient 3. $E M \times 10000$. 


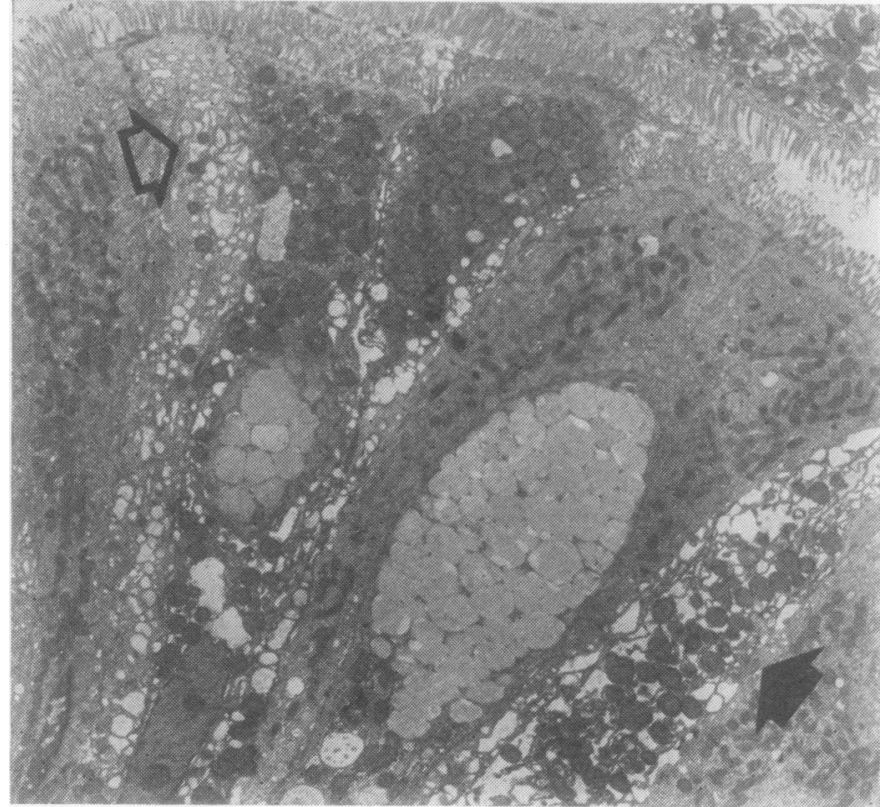

Fig. 5 Relatively normal cells are adjacent to cells which show extensive cytoplasmic vacuolation (open arrow) or necrotic degeneration (solid arrow) in patient 2. $E M \times 3000$.

distention of the LBIS was either absent or much less apparent. Intracellular vacuoles and necrotic cells were rarely seen.

\section{Discussion}

In interpreting histological abnormalities present in the small bowel of children with ALL, it should be borne in mind that various factors, other than MTX treatment, have been reported to be associated with villus damage. For example, villus shortening has been noted in a number of extraintestinal malignancies, ${ }^{11}$ although in a necropsy study of children with ALL, villus atrophy was not a feature. ${ }^{12}$ Moreover, it is unlikely that leukaemia per se was the causal factor as all our patients were in remission and in some (group B) jejunal histology was quite normal. Folate deficiency which may alter villus structure ${ }^{13}$ was not present, nor had other cytotoxic drugs (which might cause damage) been given to any of our patients for at least one week before biopsy. The contention that MTX alone was responsible is further supported by the relation between drug administration and, to a lesser extent, its total dose, and the degree of morphological damage (Table and Fig. 2).

Several animal studies have demonstrated the effect of prolonged or high doses of MTX on the small intestine ${ }^{14-16}$ and in these, subtotal villus atrophy was a prominent feature. A single in- travenous dose of MTX was found to cause ultrastructural changes in the small intestine of adults with a variety of malignancies or with psoriasis. $17 \stackrel{\mathbb{Q}}{\Omega}$ These consisted of cytoplasmic vacuolation, patchy $\overrightarrow{\overrightarrow{0}}$ degeneration and fragmentation of the microvilli. $\exists$ Similar alterations have been described in adults with solid tumours who have received various combina-? tions of chemotherapy. ${ }^{18}$ Such degenerative changes resemble those seen in the pre-extrusion cells normally found towards the tip of the villus. ${ }^{1019}$ It 3 is possible therefore, that MTX blocks enterocyte generation within the crypts resulting in the ageing of $\frac{\circ}{3}$ cells along the shaft of the villus (middle region) rather than within the distal extrusion zone. Prolonged exposure to MTX may lead to shedding of $\frac{D}{0}$ these cells with consequent denudation of the villus surface. This might be the basis of severe clinical $\tilde{N}^{\circ}$ toxicity in man and of the villus atrophy reported in $N$ laboratory animals given large doses of MTX.

A predominant feature in MTX-treated patients with ALL was the markedly enlarged LBIS with enterocyte atrophy. A similar morphological change has been reported in animals during water absorp- $\mathbb{D}$ tion $^{20}$ and following in vitro incubation of strips of rat small gut in hypotonic saline. ${ }^{21}$ Apart from premedication, our patients were not given fluids $\stackrel{\mathbb{D}}{\circ}$ immediately before biopsy, and although this was $\frac{?}{\mathbb{8}}$ common to all those studied, the abnormalities $\varrho$ described were present only in group A patients. It may be that these changes are due to a metabolic 8 
derangement causing an alteration either in intercellular binding or in fluid transport resulting in an accumulation of fluid between the enterocytes.

In this study, two of the three patients given MTX within $3 \mathrm{~h}$ of biopsy, showed some degree of structural damage (Table). This suggests that, in addition to crypt cell generation, there is a direct toxic effect also on mature enterocytes. This may be related to the effect of the drug, in non-dividing cells, ${ }^{22}{ }^{23}$ upon RNA and protein synthesis. A very early in vitro effect of MTX on enterocyte oxygen consumption has been described in animal studies. ${ }^{24}$ At the other extreme, the relative absence of damage $96 \mathrm{~h}$ after a single dose of MTX is consistent with the normal cell turnover time in jejunum. ${ }^{25}$

The variation in the degree of damage between cells on the same villus was a striking feature. In places, normal enterocytes were adjacent to markedly atrophic or necrotic cells (Fig. 5). These variations may be caused by local differences in DNA or RNA, the concentrations of dihydrofolate reductase or concentrations of folic acid intermediary metabolites at the time of MTX administration.

Whatever the pathogenesis of these changes, it would appear that even in the absence of toxic side-effects, therapeutic doses of MTX may cause alterations in villus epithelial architecture which may account for some of the pathophysiological abnormalities already described. ${ }^{3} 5$

We are grateful for valuable technical assistance to Dr S Cameron, Mr T McLaughlin, Mr J Reid and the staffs of the Electron Microscopy and Histopathology Laboratories of the Royal Victoria Hospital.

CRP is in receipt of a Royal Belfast Hospital for Sick Children Research Fellowship.

\section{References}

${ }^{1}$ Farber S, Cutler EC, Hawkins JW, Harrison JH, Pierce EC, Lenz GG. The action of pteroylglutamic acid conjugates on man. Science 1947;106:619-21.

2 Taylor SG, Hass GM, Crumrine JL, Slaughter DP. Toxic reactions of 4-amino-pteroylglutamic acid (aminopterin) in patients with far advanced neoplastic disease. Cancer 1950;3:493-503.

${ }^{3}$ Craft AW, Kay HEM, Lawson DN, McEwain TL. Methotrexate-induced malabsorption in children with acute lymphoblastic leukaemia. Br Med J 1977;ii:15112.

4 Freeman-Narrod M. The pharmacology of methotrexate. In: Porter R, Wiltshaw $\mathrm{E}$, eds. Methotrexate in the treatment of cancer. Baltimore: Williams and Wilkins, 1962:17-23.

${ }^{5}$ Smith DK, Omura GA, Ostroy F. Clinical pharmacology of intermediate dose oral methotrexate. Cancer Chemother Pharmacol 1980;4:117-20.

- Kearney PJ, Light PA, Preece A, Mott MG. Unpredictable serum levels after oral methotrexate in children with acute lymphoblastic leukaemia. Cancer Chemother Pharmacol 1979;3:117-120.

${ }^{7}$ Pinkerton CR, Welshman SG, Bridges JM, Glasgow JFT. Absorption of methotrexate under standardised conditions in children with acute lymphoblastic leukaemia. Br J Cancer 1980;42:613-5.

${ }^{8}$ Glasgow JFT, Corkey CWB, Molla A. Critical assessment of small bowel biopsy in children. Arch Dis Child 1979;54: 604-8.

${ }^{9}$ Dunnill MS, Whitehead R. A method for the quantitation of small intestinal biopsy specimens. J Clin Pathol 1972; 25:243-6.

${ }^{10}$ Philips AD, France NE, Walker-Smith JA. The structure of the enterocyte in relation to its position on the villus in childhood: an electron microscopical study. Histopathology 1979;3:117-30.

11 Deller DJ, Murrell TGC, Blowes R. Jejunal biopsy in malignant disease. Aust Ann Med 1967;16:236-41.

12 Weston JT, Guin GH. Epithelial atypias with chemotherapy in 100 acute childhood leukaemias. Cancer 1955;8:17986.

${ }^{13}$ Hermos JA, Adams WH, Liu YK, Sullivan LW, Trier JS. Mucosa of the small intestine in folate deficient alcoholics. Ann Intern Med 1972;76:957-65.

${ }^{14}$ Altmann GG. Changes in the mucosa of the small intestine following methotrexate administration or abdominal $x$-irradiation. Am J Anat 1974;140:263-71.

${ }^{15}$ Baskerville A, Batter-Hatton D. Intestinal lesions induced experimentally by methotrexate. Br J Exp Pathol 1977; $58: 663-9$.

16 Jeynes BJ, Altmann GG. Light and scanning electron microscopic observations of the effects of sublethal doses of methotrexate on the rat small intestine. Anat Rec $1978 ; 191: 1-18$.

17 Trier JS. Morphological alterations induced by methotrexate in the mucosa of human proximal intestine. Gastroenterology 1962;43:407-24.

${ }^{18}$ Shaw MT, Spector MH, Ladman AJ. Effects of cancer, radiotherapy and cytotoxic drugs on intestinal structure and function. Cancer Treat Rev 1979;6:141-51.

19 Potten CS, Allen TD. Ultrastructure of cell loss in intestinal mucosa. J Ultrastruct Res 1977;60:272-7.

${ }^{20}$ Kulenkampff $\mathbf{H}$. The structural basis of intestinal absorption. In: Forth W, Rummel W, eds. Pharmacology of intestinal absorption. Vol 1. Oxford: Pergamon Press, 1975:1-69.

${ }_{21}$ McElligott TF, Beck IT, Dinda PK, Thompson S. Correlation of structural changes at different levels of the jejunal villus with positive and negative net water transport in vivo and in vitro. Can J Physiol Pharmacol 1975;53:439-50.

${ }^{22}$ Margolis S, Philips FS, Sternberg SS. The cytotoxicity of methotrexate in mouse small intestine in relation to inhibition of folic acid reductase and of DNA synthesis. Cancer Res $1971 ; 31: 2037-46$.

${ }^{23}$ Bleyer WA. The clinical pharmacology of methotrexate. Cancer $1978 ; 41: 36-51$.

${ }^{24}$ Vitale JJ, Zamcheck N, Digiorgio J, Hegsted DM. Effects of aminopterin administration on the respiratory and morphology of the gastrointestinal mucosa of rats. J Lab Clin Med 1954;43:583-94.

${ }^{25}$ Creamer B. The turnover of the epithelium of the small intestine. Br Med Bull 1967;23:226-30.

Requests for reprints to: Dr CR Pinkerton, Department of Child Health, Institute of Clinical Science, Grosvenor Road, Belfast BT12 6BJ, N Ireland. 